\title{
Prevalence of occult pre-malignant or malignant pathology at the time of uterine morcellation for benign disease*
}

\author{
Emily C. Von Bargen ${ }^{1,2,{ }^{*}}$, Cara L. Grimes ${ }^{3}$, Kavita Mishra ${ }^{4}$, Rui Wang ${ }^{2,5}$, Miriam J. \\ Haviland $^{2,5}$, Michele R. Hacker ${ }^{2,5}$, Joseph A. Carnevale ${ }^{4}$, Alyssa J. Estes ${ }^{6}$, and Eman A. \\ Elkadry ${ }^{1,2}$ \\ ${ }^{1}$ Department of Obstetrics and Gynecology, Mount Auburn Hospital, Harvard Medical School \\ affiliate, Cambridge, MA, USA \\ ${ }^{2}$ Beth Israel Deaconess Medical Center, Harvard Medical School affiliate, Boston, MA, USA \\ ${ }^{3}$ Department of Obstetrics and Gynecology, Columbia University Medical Center, New York, NY, \\ USA \\ ${ }^{4}$ Department of Obstetrics and Gynecology, Brown University, Providence, RI, USA \\ ${ }^{5}$ Harvard Medical School, Boston, MA, USA \\ ${ }^{6}$ Touro College of Osteopathic Medicine, New York, NY, USA
}

\section{Abstract}

Objective-To determine the prevalence of occult pre-malignant or malignant uterine pathology at the time of laparoscopic surgery with open power morcellation for benign gynecologic disease.

\begin{abstract}
Methods-The present multicenter, retrospective cohort study included women who underwent open power for benign indications between January 1, 2007, and February 28, 2014, at three academic medical centers in the USA. The primary outcome was pre-malignant or malignant pathology at the time of open power morcellation, and was determined from the patients' pathology reports.
\end{abstract}

Results-During the study period, 1214 women underwent open power morcellation for benign indications Similar characteristics were observed between patients with normal pathology and those with pre-malignant or malignant uterine pathology, including body mass index, parity, hypertension, diabetes, breast cancer, and smoking (all $P>0.129$ ). Among patients who underwent open power morcellation, 14 (1.2\%) had occult pre-malignant or malignant pathology; $5(0.4 \%)$

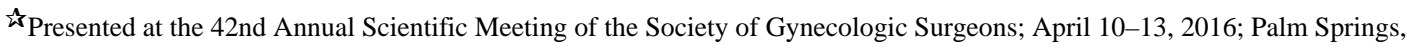
California, USA.

"Correspondence: Emily C. Von Bargen, Massachusetts General Hospital, Vincent Obstetrics and Gynecology, Female Pelvic Medicine and Reconstructive Surgery, Boston, MA, USA. evonbargen@ partners.org.

AUTHOR CONTRIBUTIONS

ECVB, KM, RW, JAC, and AJE contributed to the study design, data collection and interpretation, and to the writing and revision of the manuscript. CLG and EAE contributed to the study design, data interpretation, and to the writing and revision of the manuscript. MJH and MRH contributed to the study design, data analysis and interpretation, and to the writing and revision of the manuscript. All authors approved the final manuscript.
}

CONFLICT OF INTEREST

The authors have no conflicts of interest. 
women had endometrial adenocarcinoma and $1(0.1 \%)$ had low-grade endometrial stromal sarcoma. There were eight patients with malignant pathology who underwent additional surgical exploration and were disease free at their final clinical visit, with a median follow-up time of 42.0 months (interquartile range 5.0-62.0 months).

Conclusion-Endometrial adenocarcinoma and low-grade endometrial stromal sarcoma were rare in the present study and there were no reports of leiomyosarcoma.

\section{Keywords}

Fibroids; Hysterectomy; Morcellation; Myomectomy; Uterine malignancy

\section{INTRODUCTION}

Hysterectomy is one of the most common surgeries performed in the USA. ${ }^{1}$ Indications can range from benign causes to gynecologic malignancy, with leiomyoma and abnormal uterine bleeding two of the most frequent indications. ${ }^{2}$

In the USA, uterine cancer is the most common gynecologic malignancy, with approximately $54-870$ new cases in $2015 .{ }^{3}$ Uterine sarcomas are uncommon, accounting for approximately $3 \%$ of malignant uterine tumors; ${ }^{4}$ however, they are highly aggressive and can be difficult to differentiate from benign myomas. Further, an endometrial biopsy is less reliable in detecting uterine sarcoma compared with uterine epithelial tumors because sarcomas originate in the myometrium. ${ }^{5}$

As minimally invasive gynecologic surgery permits faster recovery, shorter hospital stay, and reduced postoperative pain, it is the preferred method for performing myomectomies and hysterectomies. ${ }^{6}$ To facilitate removal of extirpated tissue during minimally invasive surgery, the uterus or a myoma is cut into small pieces (morcellated), allowing the specimen to be removed through smaller incisions. The power morcellator is an electromechanicallyassisted instrument with a rotating blade that cuts the specimen into fragments at the time of the procedure. Morcellation can be performed in either a contained environment using a retrieval bag (closed) or uncontained (open) within the abdominal cavity.

In November 2014, the FDA stipulated that power morcellation should not be used for "the majority of women" undergoing uterine fibroid surgery owing to the potential risk of spreading occult malignancy. ${ }^{7}$ Based on a literature review, the statement concluded that, among women undergoing a hysterectomy or myomectomy for fibroids, the prevalence of uterine sarcoma and unsuspected uterine leiomyosarcoma were 1 in 352 and 1 in 498, respectively. ${ }^{7}$ Following the FDA statement on power morcellation, Pritts et al. ${ }^{8}$ performed a meta-analysis of 133 studies where surgery was performed for myomas and patient histopathology was known; the prevalence of leiomyosarcoma was approximately 1 in 2000 $(0.05 \%)$ surgeries and was further reduced to 1 in 8300 surgeries when the analysis was restricted to 64 prospective studies. ${ }^{8}$ systematic From these two reviews, the reported prevalence of leiomyosarcoma is within the range 1 in $498-8300$ hysterectomies or myomectomies performed for myoma. Other concerns related to open power morcellation include that, if an undiagnosed uterine malignancy is present, accurate surgical staging is 
difficult and the lack of management and optimal treatment guidelines for these patients presents further challenges. ${ }^{9}$

The objective of the present study was to quantify the prevalence of pre-malignant and malignant uterine pathology among women undergoing hysterectomy or myomectomy for benign disease with open power morcellation, and to describe long-term outcomes and management among these patients. It was hypothesized that a low prevalence of unexpected uterine pathology would be recorded at the time of surgical treatment for benign disease among women undergoing open power morcellation.

\section{MATERIALS AND METHODS}

The present multicenter, retrospective cohort study included a sample of convenience, including all women aged at least 18 years who underwent open power morcellation during minimally invasive (laparoscopic or robotic) hysterectomy or myomectomy for benign indications between January 1, 2007, and February 28, 2014. The chart review included data from three academic medical centers: Mount Auburn Hospital, Cambridge, Massachusetts, USA; Women and Infants Hospital, Providence, Rhode Island, USA; Columbia University Medical Center, New York, New York, USA. Benign indications were defined as leiomyomas, abnormal uterine bleeding, uterovaginal prolapse, or chronic pelvic pain. Eligible patients where identified using billing data for the morcellator and operative reports were reviewed to ensure that power morcellation had been used. Pathology reports were also reviewed. Patients who had malignant uterine or cervical pathology noted prior to surgery were excluded. The institutional review boards of all the study centers approved the present study and waived obtaining informed consent from participants.

Demographic, preoperative, intraoperative, and postoperative data were retrieved from patient medical records. Women were considered postmenopausal if this was documented in their medical records; all other patients were considered premenopausal. A patient was considered to have abnormal uterine bleeding if a history of menorrhagia, intermenstrual bleeding, or postmenopausal bleeding was recorded in clinical or operative notes.

The primary outcome was the presence of pre-malignant or malignant pathology at the time of open power morcellation during minimally invasive hysterectomy or myomectomy. Secondary outcomes were the presence of potentially predictive preoperative clinical factors of occult malignancy and long-term outcomes in patients who underwent open power morcellation. Continuous data not normally distributed were compared using the Wilcoxon rank-sum test, and categorical data were compared using the $\chi^{2}$ and Fisher exact tests. Data were presented as medians with interquartile ranges or numbers and percentages. Data were stored using Research Electronic Data Capture (REDCap) ${ }^{10}$ and were analyzed using SAS version 9.4 (SAS institute, Cary, NC, USA).

\section{RESULTS}

During the study period, 1214 women underwent open power morcellation for benign indications-580 (47.8\%) for leiomyoma, $503(41.4 \%)$ for abnormal uterine bleeding, 435 (35.8\%) for uterovaginal prolapse, 248 (20.4\%) for chronic pelvic pain, 22 (1.8\%) for 
endometriosis, and 41 (3.4\%) for other indications Of these patients, 509 (41.9\%) had more than one indication for surgery. There were 1044 (86.0\%) patients who underwent laparoscopic supracervical hysterectomy, 63 (5.2\%) total laparoscopic hysterectomy, 6 $(0.5 \%)$ laparoscopic assisted vaginal hysterectomy, and 101 (8.3\%) underwent laparoscopic myomectomy. There were 410 (33.8\%) patients with uterovaginal prolapse as the surgical indication who underwent concomitant sacrocolpopexy. In the study cohort, $14(1.2 \%)$ patients had pre-malignant or malignant uterine pathology; of these patients, $6(43 \%)$ had endometrial hyperplasia and $8(57 \%)$ had occult uterine malignancy, corresponding to prevalence rates of $0.5 \%$ and $0.7 \%$ for pre-malignant pathology and malignant pathology, respectively. Preoperative characteristics of women with pre-malignant or malignant pathology were similar to those of patients with normal pathology, including body mass index, parity, hypertension, diabetes, breast cancer, and smoking (all $P>0.129$ ) (Table 1).

Among the six patients with pre-malignant pathology, there were $3(50 \%)$ with simple hyperplasia without atypia, 1 (17\%) with simple atypical hyperplasia, $1(17 \%)$ with complex hyperplasia without atypia, and $1(17 \%)$ with endometrial intraepithelial neoplasia. The indications for hysterectomy among these patients included $3(50 \%)$ patients with uterovaginal prolapse, and $3(50 \%)$ with abnormal uterine bleeding and leiomyomas. There were 4 (67\%) patients with pre-malignant pathology who had a negative preoperative endometrial biopsy and 4 (67\%) who had a pelvic ultrasonography examination that showed an endometrial stripe thinner than $5 \mathrm{~mm}$.

Of the eight patients with occult uterine malignancy, 5 (63\%) had endometrial adenocarcinoma, corresponding to an overall prevalence rate of $0.4 \%$ within the whole study cohort. Of these eight patients, 1 (13\%) had a low-grade endometrial stromal sarcoma, 1 (13\%) had a uterine tumor resembling an ovarian sex cord tumor, and $1(13 \%)$ had atypical leiomyoma, equivalent to an overall prevalence of $0.1 \%$ for each, respectively (Table 2). There were no occurrences of leiomyosarcoma in the study cohort and endometrioid adenocarcinoma of the ovary was identified in one patient; the ovary was not morcellated and the patient underwent repeat surgical exploration and intraperitoneal chemotherapy.

Of the eight patients who were found to have an occult malignancy, 4 (50\%) had a benign preoperative endometrial biopsy and $4(50 \%)$ did not have a preoperative endometrial biopsy; Three patients underwent preoperative ultrasonography examination. Of the patients who had occult malignancy, 6 (75\%) underwent surgery for fibroids (constituting $1.0 \%$ of all patients with fibroids) and 2 (25\%) underwent surgery for uterovaginal prolapse (constituting $0.5 \%$ of all patients undergoing surgery for uterovaginal prolapse). There were $5(63 \%)$ patients with occult malignancy aged younger than 50 years, $3(38 \%)$ aged 51-60 years, and none older than 65 years (Table 2).

All patients who were found to have malignant pathology underwent surgical re-exploration and a second operative staging procedure. Of the five patients with initial pathologic findings of endometrial adenocarcinoma of the uterus, $3(60 \%)$ were assessed as having a grade 1 tumor, 1 (20\%) a grade 2 tumor, and 1 (20\%) a grade 3 tumor (with serous features) (Table 2). No residual tumor or tumor cells were found in these patients. Following surgical staging, two patients underwent neo-adjuvant therapy, as dictated by the initial pathology 
results. The patient with a grade 2 endometrial adenocarcinoma underwent radiation therapy, and the patient with a grade 3 endometrial adenocarcinoma with serous features underwent chemotherapy and vaginal cuff radiation.

The patient with a uterine tumor resembling an ovarian sex cord tumor underwent a second staging procedure with benign pathology. The patient with a low-grade endometrial stromal sarcoma also underwent a second operative procedure and a low-grade endometrial stromal sarcoma of the right adnexa was found. In the patient who had an atypical leiomyoma, this was found to occur in a myomectomy specimen; a total abdominal hysterectomy was performed and benign uterine pathology was found. No further treatment was necessary for these patients (Table 2).

All eight patients who had malignant pathology were disease free as of the final follow-up appointments. The median length of follow-up was 42.0 months (interquartile range 5.062.0 months). All patients except for one woman had at least 12 months of follow-up data following morcellation for an occult malignancy.

\section{DISCUSSION}

In the present large cohort of women undergoing open power morcellation for benign indications, the prevalence of pre-malignant and malignant pathology were approximately 1 in $202(0.5 \%)$ and 1 in $152(0.7 \%)$, respectively. The prevalence rates of endometrial adenocarcinoma and low-grade endometrial stromal sarcoma were $0.4 \%$ and $0.1 \%$, respectively. No patients in the cohort experienced leiomyosarcoma. No potential risk factors for pre-malignant or malignant uterine pathology were identified, although the small number of patients with abnormal uterine pathology limited the power of the present study to detect potentially meaningful associations. Further, no difference was found in the prevalence of pre-malignant or malignant pathology when patients were stratified by surgical indication.

Pre-malignant and malignant pathology were recorded in the present cohort, despite a benign preoperative endometrial biopsy and/or unremarkable pelvic ultrasound. The sensitivity of an endometrial biopsy to detect endometrial cancer is $94 \%$ with a specificity of $99 \% .{ }^{11}$ If pelvic ultrasonography demonstrates an endometrial stripe thicker than $5 \mathrm{~mm}$, the sensitivity and specificity for detecting endometrial cancer are 55\% and $97 \%$, respectively. ${ }^{11}$ These findings highlight the clinical predicament arising from imperfect sensitivity and specificity in the preoperative evaluation of women undergoing hysterectomy for presumed benign disease.

Einstein et al. ${ }^{12}$ recommended that surgical exploration was repeated in all patients found to have a malignancy in the morcellated specimen. Of the eight patients with occult uterine malignancy in the present cohort, $1(13 \%)$ was surgically upstaged after the diagnosis of low-grade endometrial stromal sarcoma in the right adnexa. These findings were similar to those of Einstein et al. ${ }^{12}$ who reported that $15 \%$ of patients were upstaged following repeat surgical exploration. The present findings were also supported by a study by Bojahr et al., ${ }^{13}$ in their large population-based prospective registry study of 10-731 morcellated uteri during 
laparoscopic supracervical hysterectomy, seven of the eight patients with endometrial carcinoma (overall rate of $0.07 \%$ ) underwent repeat surgery and no recurrences were reported over a mean follow-up time of 74 months. ${ }^{13}$ In their study, there were six sarcomas (prevalence rate $0.06 \%$ ) and all patients underwent re-peat surgical exploration. ${ }^{13}$

In the present study, the finding of a uterine malignancy after open power morcellation did not significantly change the clinical prognosis and no instances of morcellated leiomyosarcoma were detected. All women with malignant pathology were without evidence of disease and seven of the eight women had at least 1 year of follow-up data available. However, it is suggest that preoperative counseling should include discussing the rare possibility of an unanticipated pre-malignancy or malignancy, risk-to-benefit ratios, and alternative treatments when considering the use of open power morcellation. Additionally, newer closed systems could reduce the theoretical risk of disease spread, particularly in rare cases of leiomyosarcoma.

The results from the present study build on the paucity of data regarding open power morcellation, and the prevalence and long-term survival in women who experience an unexpected uterine malignancy. These data suggest a good prognosis after open power morcellation of malignant uterine tumors and, at most recent follow-up, all such patients were free from disease. The absence of leiomyosarcoma among 1214 patients reflects its rarity among women undergoing hysterectomy or myomectomy for benign indications. However, leiomyosarcoma is inherently a more aggressive carcinoma with a poorer prognosis and therefore is of greater concern if power morcellated in an open environment inadvertently. Further investigation will be necessary to more accurately predict these aggressive tumors in women contemplating hysterectomy.

Strengths of the present study included a relatively large sample size and the inclusion of patients from multiple medical centers. Further, the long-term mean follow-up time of 42.0 months, in which all women were without a recurrence of disease, was a strength.

Additionally, the sample was not restricted to a particular hysterectomy or myomectomy indication, improving the generalizability of the data. Women who had a hysterectomy or myomectomy performed via an open abdominal or vaginal approach were excluded to determine the risk of pre-malignant and malignant uterine pathology at the time of open power morcellation. The limitations included those inherent to the retrospective design, including only being able to assess symptoms and findings documented in medical charts. Given its rarity, leiomyosarcoma was not detected in any patients and a larger sample size could have generated a more accurate assessment of the prevalence of this rare malignancy.

In the present large cohort of women undergoing open power morcellation for benign indications, pre-malignant and malignant uterine pathology were rare. Leiomyosarcoma was not detected in any patients and no potential risk factors for abnormal uterine pathology were identified. Given this, improved diagnostic methods are needed to help differentiate pre-malignant/malignant and benign uterine pathology. Further studies are needed to investigate and determine more accurate methods for preoperative evaluation prior to hysterectomy or myomectomy. The power morcellator has reduced the morbidity associated with open surgery ${ }^{14,15}$ and this technique should not be abandoned altogether; rather, efforts 
should continue to refine methods for mitigating risks, such as effective containment of specimens and enhancing the clinical diagnosis of pre-malignant and malignant uterine pathology. These refinements, in the setting of strict guidelines and clinical recommendations for the use of power morcellation, will allow the continuation of providing safe minimally invasive surgical approaches for patients.

\section{Acknowledgments}

The present study was conducted with support from Harvard Catalyst, The Harvard Clinical and Translational Science Center (National Center for Research Resources and the National Center for Advancing Translational Sciences, National Institutes of Health Award UL1 TR001102), and financial contributions from Harvard University and its affiliated academic healthcare centers.

\section{References}

1. Wu JM, Wechter ME, Geller EJ, Nguyen TV, Visco AG. Hysterectomy rates in the United States, 2003. Obstet Gynecol. 2007; 110:1091-1095. [PubMed: 17978124]

2. Wright JD, Herzog TJ, Tsui J, et al. Nationwide trends in the performance of inpatient hysterectomy in the United States. Obstet Gynecol. 2013; 122:233-241. [PubMed: 23969789]

3. Siegel RL, Miller KD, Jamal A. Cancer statistics, 2015. CA Cancer J Clin. 2015; 65:5-29. [PubMed: 25559415]

4. D’Angelo E, Prat J. Uterine sarcomas: A review. Gynecol Oncol. 2010; 116:131-139. [PubMed: 19853898]

5. Bansal N, Herzog TJ, Burke W, Cohen CJ, Wright JD. The utility of preoperative endometrial sampling for detection of uterine sarcomas. Gynecol Oncol. 2008; 110:43-48. [PubMed: 18445505]

6. Johnson N, Barlow D, Lethaby A, Tavender E, Curr E, Garry R. Surgical approach to hysterectomy for benign gynaecological disease. Cochrane Database Syst Rev. 2006; (2):CD003677. [PubMed: 16625589]

7. US Food and Drug Administration. Medical Devices Safety Communications. [Accessed December 7, 2015] UPDATED Laparoscopic Uterine Power Morcellation in Hysterectomy and Myomectomy: FDA Safety Communication. Published 2014. http://www.fda.gov/medicalde-vices/safety/ alertsandnotices/ucm424443.htm

8. Pritts E, Vaness D, Berek J, et al. The prevalence of occult leiomyosarcoma at surgery for presumed uterine fibroids: A meta-analysis. Gynecol Surg. 2015; 12:165-177. [PubMed: 26283890]

9. Rivard C, Salhadar A, Kenton K. New challenges in detecting, grading and staging endometrial cancer after uterine morcellation. J Minim Invasive Gynecol. 2012; 19:313-316. [PubMed: 22417903]

10. Harris P, Taylor R, Thielke R, Payne J, Gonzalez N, Conde J. Research electronic data capture (REDCap) -A metadata-driven methodology and workflow process for providing translational research informatics support. J Biomed Inform. 2009; 42:377-381. [PubMed: 18929686]

11. Havrilesky LJ, Maxwell GL, Myers ER. Cost effectiveness analysis of annual screening strategies for endometrial cancer. Am J Obstet Gynecol. 2009; 200:640e1-640.e8. [PubMed: 19380121]

12. Einstein MH, Barakat RR, Chi DS, et al. Management of uterine malignancy found incidentally after supracervical hysterectomy or uterine morcellation for presumed benign disease. Int $\mathbf{J}$ Gynecol Cancer. 2009; 18:1065-1070.

13. Bojahr B, De Wilde R, Tchartchian G. Malignancy rate of 10,731 uteri morcellated during laparoscopic supracervical hysterectomy (LASH). Arch Gynecol Obstet. 2015; 292:665-672. [PubMed: 25820974]

14. Harris JA, Swenson SW, Uppal S, et al. Practice patterns and postoperative complications before and after Food and Drug Administration Safety Communication on power morcellation. Am J Obstet Gynecol. 2016; 214:98.e1-98.e13. [PubMed: 26314519] 
15. Siedoff MT, Wheeler SB, Rutstein SE, et al. Laparoscopic hysterectomy with morcellation vs abdominal hysterectomy for presumed fibroid tumors in premenopausal women: A decision analysis. Am J Obstet Gynecol. 2015; 212:591, e1-e8. [PubMed: 25817518] 


\section{TABLE 1}

Demographic characteristics $(\mathrm{n}=1214) .{ }^{a}$

\begin{tabular}{|c|c|c|c|c|}
\hline Variable & $\begin{array}{c}\text { Pre-malignant uterine } \\
\text { pathology }(n=6)\end{array}$ & $\begin{array}{l}\text { Malignant uterine pathology } \\
(\mathbf{n}=8)\end{array}$ & $\begin{array}{c}\text { Benign uterine pathology } \\
(\mathrm{n}=1200)\end{array}$ & $P$ value \\
\hline Age, y & $53.0(51.0-55.0)$ & $48.5(46.5-54.0)$ & $48.0(43.0-55.0)$ & 0.129 \\
\hline Body mass index $b$ & $27.1(27.1-29.5)$ & $29.3(26.2-35.0)$ & $26.3(23.4-30.3)$ & 0.157 \\
\hline Race & & & & 0.568 \\
\hline Non-Hispanic white & $4(67)$ & $7(88)$ & 863 (71.9) & \\
\hline Hispanic & 0 & 0 & $43(3.6)$ & \\
\hline Non-Hispanic black & $1(17)$ & $1(13)$ & $65(5.4)$ & \\
\hline Asian or Pacific Islander & 0 & 0 & $24(2.0)$ & \\
\hline Other or unknown & $1(17)$ & 0 & $205(17.1)$ & \\
\hline Parity & & & & 0.860 \\
\hline 0 & $1(17)$ & $3(38)$ & $251(20.9)$ & \\
\hline 1 & $1(17)$ & $1(13)$ & $160(13.3)$ & \\
\hline 2 & $4(67)$ & $4(50)$ & $744(62.0)$ & \\
\hline Unknown & 0 & 0 & $45(3.8)$ & \\
\hline History of smoking & & & & 0.331 \\
\hline Yes & $3(50)$ & $3(38)$ & $265(22.1)$ & \\
\hline No & $3(50)$ & $5(63)$ & $880(73.3)$ & \\
\hline Unknown & 0 & 0 & $55(4.6)$ & \\
\hline History of breast cancer & & & & $>0.99$ \\
\hline Yes & 0 & 0 & $29(2.4)$ & \\
\hline No & $6(100)$ & $8(100)$ & $1133(94.4)$ & \\
\hline Unknown & 0 & 0 & $38(3.2)$ & \\
\hline Menopausal status & & & & 0.910 \\
\hline Premenopausal & $4(67)$ & $6(75)$ & $867(72.3)$ & \\
\hline Postmenopausal & $2(33)$ & $2(25)$ & $320(26.7)$ & \\
\hline Unknown & 0 & 0 & $13(1.1)$ & \\
\hline Diabetes & & & & 0.530 \\
\hline Yes & $1(17)$ & 0 & $53(4.4)$ & \\
\hline No & $5(83)$ & $8(100)$ & 1111 (92.6) & \\
\hline Unknown & 0 & 0 & $36(3.0)$ & \\
\hline Hypertension & & & & 0.419 \\
\hline Yes & $2(33)$ & $3(38)$ & $223(18.6)$ & \\
\hline No & $4(67)$ & $5(63)$ & 943 (78.6) & \\
\hline Unknown & 0 & 0 & $34(2.8)$ & \\
\hline
\end{tabular}




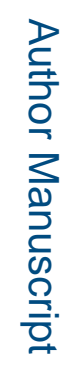

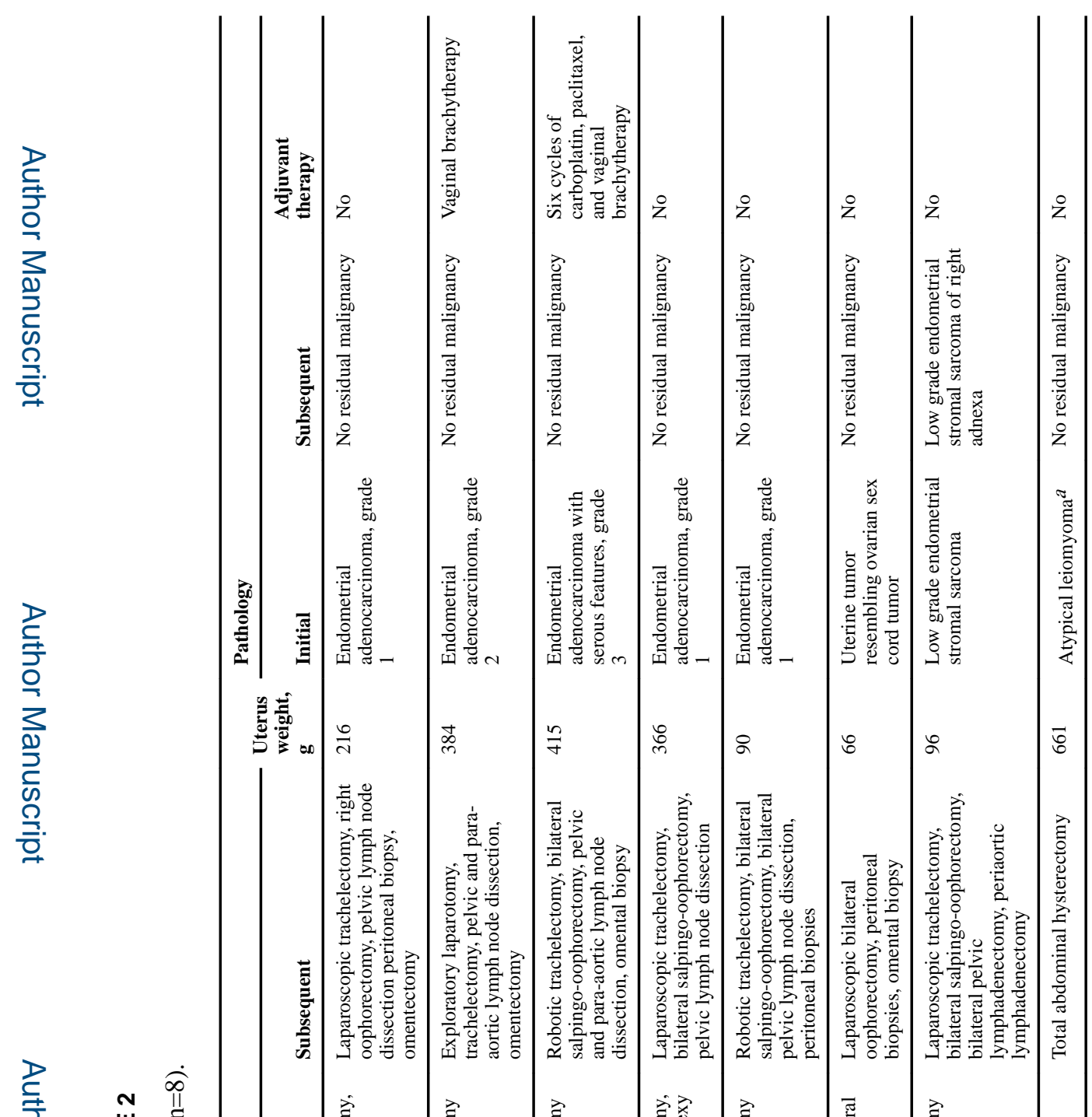

量

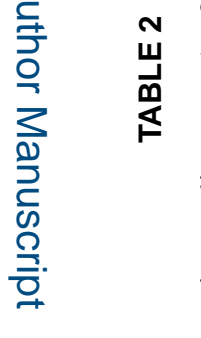

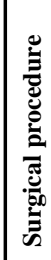
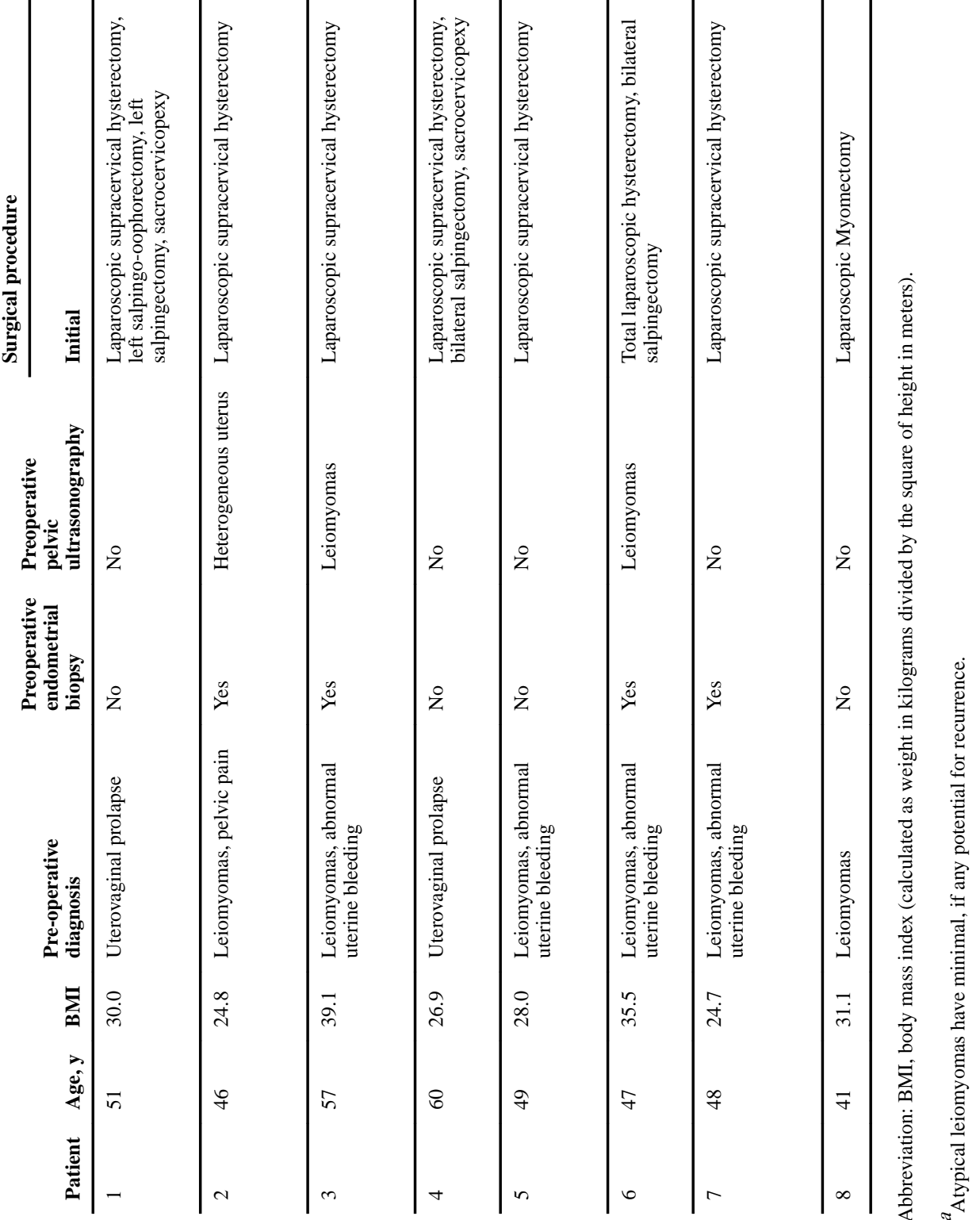